\title{
A characterization of nilpotent orbit closures among symplectic singularities
}

\section{Yoshinori Namikawa}

Symplectic singularities have been playing important roles both in algebraic geometry and geometric representation theory ever since Beauville introduced their notion in [Be]. Most examples of symplectic singularities admit natural $\mathbf{C}^{*}$-actions with only positive weights. Kaledin $[\mathrm{Ka}]$ conjectured that any symplectic singularity admits such a $\mathbf{C}^{*}$ action.

If a symplectic singularity has a $\mathbf{C}^{*}$-action with positive weights, it can be globalized to an affine variety with a $\mathbf{C}^{*}$-action. Such an affine variety is called a conical symplectic variety. More precisely, an affine normal variety $X=\operatorname{Spec} R$ is a conical symplectic variety if

(i) $R$ is positively graded: $R=\oplus_{i \geq 0} R_{i}$ with $R_{0}=\mathbf{C}$;

(ii) the smooth part $X_{\text {reg }}$ admits a homogeneous symplectic 2-form $\omega$ and it extends to a holomorphic 2 -form on a resolution $\tilde{X}$ of $X$.

Denote the $\mathbf{C}^{*}$-action by $t: X \rightarrow X\left(t \in \mathbf{C}^{*}\right)$. By the assumption we have $t^{*} \omega=t^{l} \omega$ for some integer $l$. This integer $l$ is called the weight of $\omega$ and is denoted by $w t(\omega)$. By the extension property (ii) we have $\omega t(\omega)>0$ (cf. [Na 3], Lemma (2.2)).

Let $\left\{x_{0}, \ldots, x_{n}\right\}$ be a set of minimal homogeneous generators of the $\mathbf{C}$-algebra $R$ and put $a_{i}:=\operatorname{deg} x_{i}$. We put $N:=\max \left\{a_{0}, \ldots, a_{n}\right\}$ and call $N$ the maximal weight of $X$. It is uniquely determined by a conical symplectic variety $X$. By [Na 1], there are only finitely many conical symplectic varieties $(X, \omega)$ of a fixed dimension $2 d$ and with a fixed maximal weight $N$, up to an isomorphism. In this sense it would be important to classify conical symplectic varieties with maximal weight 1 . By the homogeneous generators $\left\{x_{i}\right\}$, we can embed $X$ into an affine space $\mathbf{C}^{n+1}$. In [Na 2] we treat the case where $X \subset \mathbf{C}^{n+1}$ is a complete intersection of homogeneous polynomials. The main theorem of [ $\mathrm{Na} 2]$ asserts that $(X, \omega)$ is isomorphic to the nilpotent cone $\left(N, \omega_{K K}\right)$ of a complex semisimple Lie algebra $\mathfrak{g}$ together with the Kirillov-Kostant 2-form provided that $X$ is singular. However, there are a lot of examples of maximal weight 1 which are not of complete intersection. In fact, a nilpotent orbit $O$ of a complex semisimple Lie algebra $\mathfrak{g}$ admits the Kirillov-Kostant form $\omega_{K K}$ and if its closure $\bar{O}$ is normal, then $\left(\bar{O}, \omega_{K K}\right)$ is a conical symplectic variety with maximal weight 1 by Panyushev $[\mathrm{Pa}]$ and Hinich [Hi].

A main purpose of this article is to prove that they actually exhaust all conical symplectic varieties with maximal weight 1 .

Theorem. Let $(X, \omega)$ be a conical symplectic variety with maximal weight 1 . Then $(X, \omega)$ is isomorphic to one of the following:

(i) $\left(\mathbf{C}^{2 d}, \omega_{s t}\right)$ with $\omega_{s t}=\Sigma_{1 \leq i \leq d} d z_{i} \wedge d z_{i+d}$,

(ii) $\left(\bar{O}, \omega_{K K}\right)$ where $\bar{O}$ is a normal nilpotent orbit closure of a complex semisimple Lie algebra $\mathfrak{g}$ and $\omega_{K K}$ is the Kirillov-Kostant form. 
There is a non-normal nilpotent orbit closure in a complex semisimple Lie algebra. The normalization $\tilde{O}$ of such an orbit closure $\bar{O}$ is also a conical symplectic variety 11 But the maximal weight of $\tilde{O}$ is usually larger than 12

We first notice that $\omega$ determines a Poisson structure on $X_{\text {reg }}$ in a usual way. By the normality of $X$, it uniquely extends to a Poisson structure $\{\cdot, \cdot\}: O_{X} \times O_{X} \rightarrow O_{X}$. In particular, $R$ becomes a Poisson $\mathbf{C}$-algebra with a Poisson bracket of degree $-w t(\omega)$.

In the remainder, $X$ is a conical symplectic variety with the maximal weight $N=1$. First of all, we prove in Proposition 1 that $w t(\omega)=2$ or $w t(\omega)=1$. In the first case $(X, \omega)$ is isomorphic to an affine space $\mathbf{C}^{2 d}$ together with the standard symplectic form $\omega_{s t}$. In the second case the Poisson bracket has degree -1 and $R_{1}$ has a natural Lie algebra structure. Then it is fairly easy to show that $X$ is a coadjoint orbit closure of a complex Lie algebra $\mathfrak{g}$ (Proposition 3). If $X$ has a crepant resolution, we can prove that $\mathfrak{g}$ is semisimple in the same way as in [Na 2]. But $X$ generally does not have such a resolution and we need a new method to prove the semisimplicity. This is nothing but Proposition 4.

Proposition 1 Assume that $X$ is a conical symplectic variety with maximal weight $N=1$. Then $w t(\omega)=1$ or $w t(\omega)=2$. If $w t(\omega)=2$, then $(X, \omega)$ is isomorphic to an affine space $\left(\mathbf{C}^{2 d}, \omega_{s t}\right)$ with the standard symplectic form..

Remark. As is remarked in the beginning of [Na 2, §2], if $X$ is a smooth conical symplectic variety with maximal weight 1 , then $(X, \omega) \cong\left(\mathbf{C}^{2 d}, \omega_{s t}\right)$. Hence $X$ is singular exactly when $w t(\omega)=1$.

Proof. Since $N=1$, the coordinate $\operatorname{ring} R$ is generated by $R_{1}$. We put $l:=w t(\omega)$. We already know that $l>0$. If $l>2$, then we have $\left\{R_{1}, R_{1}\right\}=0$ and hence $\{R, R\}=0$, which is absurd. We now assume that $l=2$ and prove that $X$ is an affine space with the standard symplectic form. Then the Poisson bracket induces a skew-symmetric form $R_{1} \times R_{1} \rightarrow R_{0}=\mathrm{C}$. If this is a degenerate skew-symmetric form, then we can choose a non-zero element $x_{1} \in R_{1}$ such that $\left\{x_{1}, \cdot\right\}=0$. Notice that $x_{1}=0$ determines a nonzero effective divisor $D$ on $X_{r e g}$. If we choose a general point $a \in D$, then the reduced divisor $D_{\text {red }}$ is smooth around $a$. Consider an analytic open neighborhood $U \subset X_{\text {reg }}$ of $a$. Then there is a system of local coordinates $\left\{z_{1}, \ldots, z_{2 d}\right\}$ of $U$ such that $x_{1}$ can be written as $x_{1}=z_{1}^{m}$ for a suitable $m>0$. The Poisson structure on $X$ induces a non-degenerate Poisson structure $\{\cdot, \cdot\}_{U}$ on $U$. But, by the choice of $x_{1}$, we have $\left\{z_{1}^{m}, \cdot\right\}_{U}=m z_{1}^{m-1}\left\{z_{1}, \cdot\right\}_{U}=0$, which implies that $\left\{z_{1}, \cdot\right\}_{U}=0$. This contradicts that the Poisson bracket $\{\cdot, \cdot\}_{U}$ is non-degenerate.

Therefore the skew-symmetric form is non-degenerate. In this case $X$ is a closed Poisson subscheme of an affine space with a non-degenerate Poisson structure induced by the standard symplectic form. But such an affine space has no Poisson closed subscheme

\footnotetext{
${ }^{1}$ By [K-P, Proposition 7.4] $\bar{O}$ is always resolved by a vector bundle $Y$ over $G / P$ with a parabolic subgroup $P$ of the adjoint group $G$ of $\mathfrak{g}$. Denote this resolution by $\pi: Y \rightarrow \bar{O}$. The map $\pi$ factorizes as $Y \rightarrow \tilde{O} \rightarrow \bar{O}$. The fiber $\pi^{-1}(0)$ coincides with the zero section of $Y$, which is isomorphic to $G / P$. As $G / P$ is connected, the fibre $\mu^{-1}(0)$ of the normalization map $\mu: \tilde{O} \rightarrow \bar{O}$ consists of just one point, say $x \in \tilde{O}$. The $\mathbf{C}^{*}$-action on $\bar{O}$ extends to a $\mathbf{C}^{*}$-action on $\tilde{O}$ with a unique fixed point $x$. It is easily checked that this $\mathbf{C}^{*}$-action has only positive weights and $\tilde{O}$ becomes a conical symplectic variety.

${ }^{2}$ It may happen that $\tilde{O}$ coincides with a normal nilpotent orbit closure of a different complex semisimple Lie algebra. In such a case the maximal weight is 1.
} 
except the affine space itself. Therefore $X=\operatorname{Spec} R$. Q.E.D.

The regular part $X_{r e g}$ of a conical symplectic variety $X$ is a smooth Poisson variety. Let $\Theta_{X_{r e g}}$ denote the sheaf of vector fields on $X_{r e g}$. By using the Poisson bracket we define the Lichnerowicz-Poisson complex

$$
0 \rightarrow \Theta_{X_{\text {reg }}} \stackrel{\delta_{1}}{\rightarrow} \wedge^{2} \Theta_{X_{\text {reg }}} \stackrel{\delta_{2}}{\rightarrow} \ldots
$$

by

$$
\begin{aligned}
& \delta_{p} f\left(d a_{1} \wedge \ldots \wedge d a_{p+1}\right):=\sum_{i=1}^{p+1}(-1)^{i+1}\left\{a_{i}, f\left(d a_{1} \wedge \ldots \hat{d a_{i}} \wedge \ldots \wedge d a_{p+1}\right\}\right. \\
& +\sum_{j<k}(-1)^{j+k} f\left(d\left\{a_{j}, a_{k}\right\} \wedge d a_{1} \wedge \ldots \wedge d \hat{a}_{j} \wedge \ldots \wedge d \hat{a}_{k} \wedge \ldots \wedge d a_{p+1}\right) .
\end{aligned}
$$

In the Lichnerowicz-Poisson complex, $\wedge^{p} \Theta_{X_{\text {reg }}}$ is placed in degree $p$. The LichnerowiczPoisson complex of $X_{\text {reg }}$ is closely related to the Poisson deformation of $(X,\{\}$,$) . For$ details, see [ $\mathrm{Na} 4]$.

In the remainder we assume that $w t(\omega)=1$. The Poisson bracket then defines a pairing map $R_{1} \times R_{1} \rightarrow R_{1}$ and $R_{1}$ becomes a Lie algebra. We denote by $\mathfrak{g}$ this Lie algebra. As all generators have weight 1 , we have a surjection $\oplus \operatorname{Sym}^{i}\left(R_{1}\right) \rightarrow R$. It induces a $\mathbf{C}^{*}$-equivariant closed embedding $X \rightarrow \mathfrak{g}^{*}$.

Recall that the adjoint group $G$ of $\mathfrak{g}$ (cf. [Pro, p.86]) is defined as a subgroup of $G L(\mathfrak{g})$ generated by all elements of the form $\exp (a d v)$ with $v \in \mathfrak{g}$. The adjoint group $G$ is a complex Lie subgroup of $G L(\mathfrak{g})$, but it is not necessarily a closed algebraic subgroup of $G L(\mathfrak{g})$. Moreover, the Lie algebra $\operatorname{Lie}(G)$ does not necessarily coincide with $\mathfrak{g}$. We have $\operatorname{Lie}(G)=\mathfrak{g}$ if and only if the adjoint representation is a faithful $\mathfrak{g}$-representation, or equivalently, $\mathfrak{g}$ has trivial center.

Proposition 2. Let $\operatorname{Aut}^{\mathbf{C}^{*}}(X, \omega)$ denote the $\mathbf{C}^{*}$-equivariant automorphism group preserving $\omega$. Then the identity component of $\operatorname{Aut}^{\mathbf{C}^{*}}(X, \omega)$ can be identified with the adjoint group $G$ of $\mathfrak{g}$. Moreover $\mathfrak{g}$ has trivial center. In particular, $\mathfrak{g}$ is the Lie algebra of the linear algebraic group $\operatorname{Aut}^{\mathbf{C}^{*}}(X, \omega)$.

Proof . Let $\left(\wedge^{\geq 1} \Theta_{X_{\text {reg }}}, \delta\right)$ be the Lichnerowicz-Poisson complex for the smooth Poisson variety $X_{\text {reg }}$. The algebraic torus $\mathbf{C}^{*}$ acts on $\Gamma\left(X_{r e g}, \wedge^{p} \Theta_{X_{r e g}}\right)$ and there is an associated grading

$$
\Gamma\left(X_{r e g}, \wedge^{p} \Theta_{X_{r e g}}\right)=\oplus_{n \in \mathbf{Z}} \Gamma\left(X_{r e g}, \wedge^{p} \Theta_{X_{r e g}}\right)(n) .
$$

Since the Poisson bracket of $X$ has degree -1 , the coboudary map $\delta$ has degree -1 ; thus we have a complex

$$
\Gamma\left(X_{\text {reg }}, \Theta_{X_{r e g}}\right)(0) \stackrel{\delta_{1}}{\rightarrow} \Gamma\left(X_{\text {reg }}, \wedge^{2} \Theta_{X_{r e g}}\right)(-1) \stackrel{\delta_{2}}{\rightarrow} \ldots
$$

The kernel $\operatorname{Ker}\left(\delta_{1}\right)$ of this complex is isomorphic to the tangent space of $\operatorname{Aut}^{\mathbf{C}^{*}}(X, \omega)$ at $[i d]$. In fact, an element of $\operatorname{Ker}\left(\delta_{1}\right)$ corresponds to a derivation of $O_{X_{\text {reg }}}$ (or an infinitesimal automorphism of $X_{r e g}$ ) preserving the Poisson structure, but it uniquely 
extends to a derivation of $O_{X}$ preserving the Poisson structure (cf. [Na 4, Proposition $8])$.

The Lichnerowicz-Poisson complex $\left(\wedge^{\geq 1} \Theta_{X_{\text {reg }}}, \delta\right)$ is identified with the truncated De Rham complex $\left(\Omega_{\bar{X}_{r e q}}^{\geq 1}, d\right)$ by the symplectic form $\omega$ (cf. [Na 4, Proposition 9], [Na 3, Section 3]). The algebraic torus $\mathbf{C}^{*}$ acts on $\Gamma\left(X_{\text {reg }}, \Omega_{X_{\text {reg }}}^{p}\right)$ and there is an associated grading

$$
\Gamma\left(X_{r e g}, \Omega_{X_{r e g}}^{p}\right)=\oplus_{n \in \mathbf{Z}} \Gamma\left(X_{r e g}, \Omega_{X_{r e g}}^{p}\right)(n) .
$$

The coboundary map $d$ has degree 0 ; thus we have a complex

$$
\Gamma\left(X_{\text {reg }}, \Omega_{X_{\text {reg }}}^{1}\right)(1) \stackrel{d_{1}}{\rightarrow} \Gamma\left(X_{\text {reg }}, \Omega_{X_{\text {reg }}}^{2}\right)(1) \stackrel{d_{2}}{\rightarrow} \ldots
$$

Since $\omega$ has weight 1 , this complex is identified with the the Lichnerowicz-Poisson complex above.

There is an injective map $d: \Gamma\left(X_{\text {reg }}, O_{X_{\text {reg }}}\right)(1) \rightarrow \Gamma\left(X_{\text {reg }}, \Omega_{X_{\text {reg }}}^{1}\right)(1)$.

We shall prove that $\operatorname{Ker}\left(d_{1}\right)=\Gamma\left(X_{\text {reg }}, O_{X_{\text {reg }}}\right)(1)$. The $\mathbf{C}^{*}$-action on $X$ defines a vector field $\zeta$ on $X_{\text {reg }}$. For $v \in \Gamma\left(X_{\text {reg }}, \Omega_{X_{\text {reg }}}^{1}\right)(1)$, the Lie derivative $L_{\zeta} v$ of $v$ along $\zeta$ equals $v$. If moreover $v$ is $d$-closed, then one has $v=d\left(i_{\zeta} v\right)$ by the Cartan relation

$$
L_{\zeta} v=d\left(i_{\zeta} v\right)+i_{\zeta}(d v)
$$

This means that $v \in \Gamma\left(X_{r e g}, O_{X_{r e g}}\right)(1)$. On the other hand, we have $\Gamma\left(X_{\text {reg }}, O_{X_{\text {reg }}}\right)(1)=$ $\Gamma\left(X, O_{X}\right)(1)=R_{1}=\mathfrak{g}$.

It follows from the identification of $\operatorname{Ker}\left(\delta_{1}\right)$ and $\operatorname{Ker}\left(d_{1}\right)$ that every element of $\operatorname{Ker}\left(\delta_{1}\right)$ is a Hamiltonian vector field $H_{f}:=\{f, \cdot\}$ for some $f \in R_{1}$. In particular, for $g \in R_{1}$, we have $H_{f}(g)=[f, g]$. Since $H_{f} \neq 0$ for a non-zero $f$, the map $a d: \mathfrak{g} \rightarrow \operatorname{End}(\mathfrak{g})$ is an injection. Notice that an element of $\operatorname{Aut}^{\mathbf{C}^{*}}(X, \omega)$ determines an automorphism of a graded $\mathbf{C}$-algebra $R$. In particular, it induces a $\mathbf{C}$-linear automorphism of $R_{1}=\mathfrak{g}$. Since $R$ is generated by $R_{1}$, this linear automorphism completely determines an automorphism of $R$. Hence, both $G$ and $\operatorname{Aut}^{\mathbf{C}^{*}}(X, \omega)$ are subgroups of $G L(\mathfrak{g})$. The tangent spaces of both subgroups at $[i d]$ coincide with $\mathfrak{g} \cong \operatorname{ad}(\mathfrak{g}) \subset \operatorname{End}(\mathfrak{g})$. Therefore $G$ is the identity component of $\operatorname{Aut}^{\mathbf{C}^{*}}(X, \omega)$ and $\operatorname{Lie}(G)=\mathfrak{g}$. Q.E.D.

Proposition 3 The symplectic variety $X$ coincides with the closure of a coadjoint orbit of $\mathfrak{g}^{*}$.

Proof. Since $G$ is the identity component of $\operatorname{Aut}^{\mathrm{C}^{*}}(X, \omega), X$ is stable under the coadjoint action of $G$ on $\mathfrak{g}$. Hence $X$ is a union of $G$-orbits. The $G$-orbits in $X$ are symplectic leaves of the Poisson variety $X$. In our case, since $X$ has only symplectic singularities, $X$ has only finitely many symplectic leaves by [Ka]. Therefore $X$ consists of finite number of $G$-orbits; hence there is an open dense $G$-orbit and $X$ is the closure of such an orbit. Q.E.D.

For the unipotent radical $U$ of $G$, let us denote by $\mathfrak{n}$ its Lie algebra 3 . Assume that $\mathfrak{n} \neq 0$. Then the center $z(\mathfrak{n})$ of $\mathfrak{n}$ is also non-trivial because $\mathfrak{n}$ is a nilpotent Lie algebra.

\footnotetext{
${ }^{3}$ The ideal $\mathfrak{n}$ is actually the nilradical of $\mathfrak{g}$ when $\mathfrak{g}$ has trivial center.
} 
Moreover $z(\mathfrak{n})$ is an ideal of $\mathfrak{g}$. In fact, it is enough to prove that, if $y \in \mathfrak{g}$ and $z \in z(\mathfrak{n})$, then $[x,[y, z]]=0$ for any $x \in \mathfrak{n}$. Consider the Jacobi identity

$$
[x,[y, z]]+[y,[z, x]]+[z,[x, y]]=0 .
$$

First, since $z \in z(\mathfrak{n})$, one has $[z, x]=0$. Next, since $\mathfrak{n}$ is an ideal of $\mathfrak{g}$, we have $[x, y] \in \mathfrak{n}$; hence $[z,[x, y]]=0$. It then follows from the Jacobi identity that $[x,[y, z]]=0$.

Proposition 4. Let $\mathfrak{g}$ be a complex Lie algebra with trivial center whose adjoint group $G$ is a linear algebraic group. Assume that $\mathfrak{n} \neq 0$. Let $O$ be a coadjoint orbit of $\mathfrak{g}^{*}$ with the following properties

(i) $O$ is preserved by the scalar $\mathbf{C}^{*}$-action on $\mathfrak{g}^{*}$;

(ii) $T_{0} \bar{O}=\mathfrak{g}^{*}$, where $T_{0} \bar{O}$ denotes the tangent space of the closure $\bar{O}$ of $O$ at the origin.

Then $\bar{O}-O$ contains infinitely many coadjoint orbits; in particular $\bar{O}$ has infinitely many symplectic leaves.

Remark. This proposition shows that $\bar{O}$ cannot have symplectic singularities. In fact, if $\bar{O}$ has symplectic singularities, it has only finitely many symplectic leaves by [Ka].

Proof of Proposition 4. By a result of Mostow [Mos] (cf. [Ho], VIII, Theorem 3.5, Theorem 4.3), $G$ is a semi-direct product of a reductive subgroup $L$ and the unipotent radical $U$. Therefore we have a decomposition $\mathfrak{g}=\mathfrak{l} \oplus \mathfrak{n}$. Take an element $\phi \in O$. Then $\phi$ is a linear function on $\mathfrak{g}$, which restricts to a non-zero function on $z(\mathfrak{n})$. In fact, if $\phi$ is zero on $z(\mathfrak{n})$, then $O \subset(\mathfrak{g} / z(\mathfrak{n}))^{*}$ and hence $\bar{O} \subset(\mathfrak{g} / z(\mathfrak{n}))^{*}$, which contradicts the assumption (ii). We put $\bar{\phi}:=\left.\phi\right|_{z(\mathfrak{n})} \neq 0$.

Notice that the adjoint group $G$ is the subgroup of $\mathrm{GL}(\mathfrak{g})$ generated by all elements of the form $\exp (a d v)$ with $v \in \mathfrak{g}$. If $v \in z(\mathfrak{n})$, then $\exp (a d v)=i d+a d v$ because $(a d v)^{2}=0$ for $v \in z(\mathfrak{n})$. Let $Z(U)$ be the identity component of the center of the unipotent radical $U$. Then one can write $Z(U)=1+a d z(\mathfrak{n})$. By the assumption, the map ad $: \mathfrak{g} \rightarrow \operatorname{End}(\mathfrak{g})$ is an injection. Now we identify $z(\mathfrak{n})$ with ad $z(\mathfrak{n})$; then one can write $Z(U)=1+z(\mathfrak{n})$ and its group law is defined by $(1+v)\left(1+v^{\prime}\right):=1+\left(v+v^{\prime}\right)$ for $v, v^{\prime} \in z(\mathfrak{n})$. Fix an element $v \in z(\mathfrak{n})$ and consider $A d_{1+v}^{*}(\phi) \in \mathfrak{g}^{*}$. Since $(1+v)^{-1}=1-v$, the adjoint action

$$
A d_{(1+v)^{-1}}: \mathfrak{l} \oplus \mathfrak{n} \rightarrow \mathfrak{l} \oplus \mathfrak{n}
$$

is defined by

$$
x \oplus y \rightarrow x \oplus(-[v, x]+y)
$$

because $(a d v)(y)=0$. By definition

$$
A d_{1+v}^{*}(\phi)(x \oplus y)=\phi\left(A d_{(1+v)^{-1}}(x \oplus y)\right)=\phi(x \oplus y)-\bar{\phi}([v, x]) .
$$

Here notice that $[v, x] \in z(\mathfrak{n})$ and hence $\phi([v, x])=\bar{\phi}([v, x])$.

Since $z(\mathfrak{n})$ is an $\mathfrak{l}$-module by the Lie bracket, we decompose it into irreducible factors $z(\mathfrak{n})=\bigoplus V_{i}$. Notice that it is the same as the irreducible decomposition of $z(\mathfrak{n})$ as a $[\mathfrak{l}, \mathfrak{l}]$-module if $[\mathfrak{l}, \mathfrak{l}] \neq 0$. In fact, the reductive Lie algebra $\mathfrak{l}$ is written as a direct sum of the semi-simple part and the center: $\mathfrak{l}=[\mathfrak{l}, \mathfrak{l}] \oplus z(\mathfrak{l})$. Since $z(\mathfrak{l})$ is an Abelian Lie algebra, $z(\mathfrak{n})$ can be written as a direct sum $\oplus V_{\alpha}$ of the weight spaces for $z(\mathfrak{l})$. The semisimple part $[\mathfrak{l}, \mathfrak{l}]$ acts on each weight space $V_{\alpha}$; hence $V_{\alpha}$ is a direct sum of irreducible 
$[\mathfrak{l}, \mathfrak{l}]$ modules. These irreducible $[\mathfrak{l}, \mathfrak{l}]$ modules are stable under the $z(\mathfrak{l})$-action and, hence are irreducible $\mathfrak{l}$-modules.

When $\operatorname{dim} V_{i}=1$ for some $i$, this $V_{i}$ is an ideal of $\mathfrak{g}$. By the assumption (i), one can write $\bar{O}=\operatorname{Spec} R$ with a graded $\mathbf{C}$-algebra $R=\oplus_{j \geq 0} R_{j}$. By (ii) we see that $R_{1}=\mathfrak{g}$. Take a generator $x$ of a 1-dimensional space $V_{i}$. Then $x$ generates a Poisson ideal $I$ of $R$ and $Y:=\operatorname{Spec}(R / I)$ is a closed Poisson subscheme of $\bar{O}$ of codimension 1. Moreover, $Y$ is stable under the $G$-action. Since $\operatorname{dim} Y$ is odd, $Y$ contains infinitely many coadjoint orbits.

In the remainder we assume that $\operatorname{dim} V_{i}>1$ for all $i$. In this case $[\mathfrak{l}, \mathfrak{l}] \neq 0$. Since $\bar{\phi} \neq 0$, we can choose an $i$ such that $\left.\phi\right|_{V_{i}} \neq 0$. We fix a Cartan subalgebra $\mathfrak{h}$ of the semisimple Lie algebra $[\mathfrak{l}, \mathfrak{l}]$ and choose a set $\Delta$ of simple roots from the root system $\Phi$. We define $\mathfrak{n}^{+}:=\bigoplus_{\alpha \in \Phi^{+}}[\mathfrak{l}, \mathfrak{l}]_{\alpha}$. Let $v_{0} \in V_{i}$ be a highest weight vector of the irreducible $[\mathfrak{l}, \mathfrak{l}]$-module $V_{i}$. Then one has $\left[v_{0}, \mathfrak{n}^{+}\right]=0$ and, in particular, $\phi\left(\left[v_{0}, \mathfrak{n}^{+}\right]\right)=0$. Moreover, we may assume that $\bar{\phi}\left(v_{0}\right) \neq 0$ by replacing $\phi$ by a suitable $A d_{g}^{*}(\phi)$ with $g \in L$. This is possible. In fact, if $A d_{g}^{*}(\bar{\phi})\left(v_{0}\right)=0$ for all $g$, then $\bar{\phi}$ is zero on the vector subspace of $V_{i}$ spanned by all $A d_{g}\left(v_{0}\right)$. But, since $V_{i}$ is an irreducible $L$-representation, such a subspace coincides with $V_{i}$. This contradicts the fact that $\left.\bar{\phi}\right|_{V_{i}} \neq 0$. Since $v_{0}$ is a highest weight vector of a non-trivial $[\mathfrak{l}, \mathfrak{l}]$-irreducible module $V_{i},\left[v_{0}, h\right]$ is a multiple of $v_{0}$ by a non-zero constant for an $h \in \mathfrak{h}$. Since $\bar{\phi}\left(v_{0}\right) \neq 0$, we also have $\bar{\phi}\left(\left[v_{0}, h\right]\right) \neq 0$ for this $h \in \mathfrak{h}$.

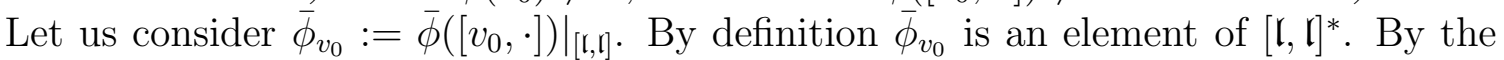
Killing form it is identified with an element of $[\mathfrak{l}, \mathfrak{l}]$. The two facts $\bar{\phi}_{v_{0}}\left(\mathfrak{n}^{+}\right)=0$ and $\bar{\phi}_{v_{0}}(h) \neq 0$ mean that $\bar{\phi}_{v_{0}}$ is not a nilpotent element of $[\mathfrak{l}, \mathfrak{l}]$.

For such $v_{0}$ and $\phi$, we consider $A d_{1+t^{-1} v_{0}}^{*}(t \phi)$, with $t \in \mathbf{C}^{*}$. One can write

$$
A d_{1+t^{-1} v_{0}}^{*}(t \phi)(x \oplus y)=t \phi(x \oplus y)-\bar{\phi}\left(\left[v_{0}, x\right]\right) .
$$

Thus one has

$$
\lim _{t \rightarrow 0} A d_{1+t^{-1} v_{0}}^{*}(t \phi)(x \oplus y)=-\bar{\phi}\left(\left[v_{0}, x\right]\right) .
$$

By definition $A d_{1+t^{-1} v_{0}}^{*}(t \phi) \in O$. Thus $\lim _{t \rightarrow 0} A d_{1+t^{-1} v_{0}}^{*}(t \phi) \in \bar{O}$. Moreover, by the equality above, we see that $\left.\lim _{t \rightarrow 0} A d_{1+t^{-1} v_{0}}^{*}(t \phi)\right|_{\mathfrak{n}}=0$; thus it can be regarded as an element of $(\mathfrak{g} / \mathfrak{n})^{*}=\mathfrak{l}^{*}$.

Furthermore, we have

$$
\left.\lim _{t \rightarrow 0} A d_{1+t^{-1} v_{0}}^{*}(t \phi)\right|_{[r, l]}=-\bar{\phi}_{v_{0}}
$$

which can be regarded as an element of $[\mathfrak{l}, \mathfrak{l}]$ by the identification $[\mathfrak{l}, \mathfrak{l}]^{*} \cong[\mathfrak{l}, \mathfrak{l}]$. As remarked above, this is not a nilpotent element.

Let us write $\mathfrak{l}$ as a direct sum of the semi-simple part and the center: $\mathfrak{l}=[\mathfrak{l}, \mathfrak{l}] \oplus z(\mathfrak{l})$. There is an $L$-equivariant isomorphism $\mathfrak{l}^{*} \cong[\mathfrak{l}, \mathfrak{l}]^{*} \oplus z(\mathfrak{l})^{*}$. Here $L$ acts trivially on the second factor $z(\mathfrak{l})^{*}$. Therefore, every coadjoint orbit of $\mathfrak{l}^{*}$ is a pair of a coadjoint orbit of $[\mathfrak{l}, \mathfrak{l}]^{*}$ and an element of $z(\mathfrak{l})^{*}$.

In our situation, we can write

$$
\bar{\phi}\left(\left[v_{0}, \cdot\right]\right)=\left.\bar{\phi}_{v_{0}} \oplus \bar{\phi}\left(\left[v_{0}, \cdot\right]\right)\right|_{z(\mathfrak{l})} .
$$


We can apply the same argument for $\lambda \phi$ with an arbitrary $\lambda \in \mathbf{C}^{*}$ to conclude that $\lambda \bar{\phi}\left(\left[v_{0}, \cdot\right]\right) \in \bar{O}$. One can write

$$
\lambda \cdot \bar{\phi}\left(\left[v_{0}, \cdot\right)=\left.\lambda \bar{\phi}_{v_{0}} \oplus \lambda \cdot \bar{\phi}\left(\left[v_{0}, \cdot\right]\right)\right|_{z(\mathfrak{l})} .\right.
$$

Since $\bar{\phi}_{v_{0}}$ is not an nilpotent element, we see that $\lambda \bar{\phi}_{v_{0}}\left(\lambda \in \mathbf{C}^{*}\right)$ are contained in mutually different coadjoint orbits of $[\mathfrak{l}, \mathfrak{l}]^{*}$.

Therefore, $\lambda \cdot \bar{\phi}\left(\left[v_{0}, \cdot\right]\right)\left(\lambda \in \mathbf{C}^{*}\right)$ are also contained in mutually different coadjoint orbits of $\mathfrak{l}^{*}$. Q.E.D.

Proof of Theorem. We already know that $w t(\omega)=1$ or $w t(\omega)=2$. In the latter case $(X, \omega)$ is isomorphic to $\left(\mathbf{C}^{2 d}, \omega_{s t}\right)$. So we assume that $w t(\omega)=1$. By Propositions 2,3 and $4,(X, \omega)$ is isomorphic to a coadjoint orbit closure $\left(\bar{O}, \omega_{K K}\right)$ of a complex reductive Lie algebra $\mathfrak{g}$ together with the Kirillov-Kostant form. Assume that $\mathfrak{g}$ is not semisimple, i.e., $\mathfrak{g}$ has non-trivial center $z(\mathfrak{g})$. In our case $O$ is preserved by the scalar $\mathbf{C}^{*}$-action on $\mathfrak{g}^{*}$. Such an orbit is contained in $(\mathfrak{g} / z(\mathfrak{g}))^{*}$. This contradicts the fact that $T_{0} \bar{O}=\mathfrak{g}^{*}$. Hence $\mathfrak{g}$ is semisimple. For a semisimple Lie algebra, a coadjoint orbit is identified with an adjoint orbit by the Killing form. A coadjoint orbit preserved by the scalar $\mathbf{C}^{*}$-action corresponds to a nilpotent orbit by this identification.

\section{References}

[Be] Beauville, A. : Symplectic singularities, Invent. Math. 139 (2000), 541-549

[Hi] Hinich, V. : On the singularities of nilpotent orbits, Israel J. Math. 73 (1991), 297-308

[Ho] Hochschild, G. P.: Basic theory of algebraic groups and Lie algebras, Graduate Texts in Mathematics 75 (1981)

[Ka] Kaledin, D.: Symplectic varieties from the Poisson point of view, J. Reine Angew. Math. 600 (2006), 135-160

[K-P] Kraft, H., Procesi, C.: On the geometry of conjugacy classes in classical groups, Comment Math. Helv. 57 (1982), 539-602

[Mos] Mostow, G.D.: Fully reducible subgroups of algebraic groups, Amer. J. Math. 78 (1956) 200-221

[Na 1] Namikawa, Y.: A finiteness theorem on symplectic singularities, Compositio Math. 152 (2016), 1225-1236

[Na 2] Namikawa, Y.: On the structure of homogeneous symplectic varieties of complete intersection, Invent. Math. 193 (2013) 159-185

[Na 3] Namikawa, Y.: Equivalence of symplectic singularities, Kyoto Journal of Mathematics, 53, No.2 (2013), 483-514

[Na 4] Namikawa, Y.: Flops and Poisson deformations of symplectic varieties, Publ. Res. Inst. Math. Sci. 44 (2008), 259 - 314

[Pa] Panyushev, D. I. : Rationality of singularities and the Gorenstein property of nilpotent orbits, Funct. Anal. Appl. 25 (1991), 225-226

[Pro] Procesi, C.: Lie groups, an approach through invariants and representations, (2007), UTX, Springer

Department of Mathematics, Graduate school of Science, Kyoto University e-mail: namikawa@math.kyoto-u.ac.jp 